\title{
Analysis of the canid Y-chromosome phylogeny using short-read sequencing data reveals the presence of distinct haplogroups among Neolithic European dogs
}

\author{
Matthew T. Oetjens ${ }^{1}$, Axel Martin ${ }^{1}$, Krishna R. Veeramah ${ }^{2}$ and Jeffrey M. Kidd ${ }^{1,3^{*}}$ (D)
}

\begin{abstract}
Background: Most genetic analyses of ancient and modern dogs have focused on variation in the autosomes or on the mitochondria. Mitochondrial DNA is more easily obtained from ancient samples than nuclear DNA and mitochondrial analyses have revealed important insights into the evolutionary history of canids. Utilizing a recently published dog Y-chromosome reference, we analyzed Y-chromosome sequence across a diverse collection of canids and determined the $Y$ haplogroup of three ancient European dogs.

Results: We identified 1121 biallelic Y-chromosome SNVs using whole-genome sequences from 118 canids and defined variants diagnostic to distinct dog $Y$ haplogroups. Similar to that of the mitochondria and previous more limited studies of $Y$ diversity, we observe several deep splits in the $Y$-chromosome tree which may be the result of retained Y-chromosome diversity which predates dog domestication or post-domestication admixture with wolves. We find that Y-chromosomes from three ancient European dogs (4700-7000 years old) belong to distinct clades.

Conclusions: We estimate that the time to the most recent comment ancestor of dog $Y$ haplogroups is 68-151 thousand years ago. Analysis of three Y-chromosomes from the Neolithic confirms long stranding population structure among European dogs.
\end{abstract}

Keywords: Canid, Y-chromosome haplogroups, Ancient dog

\section{Background}

Dogs are a domesticated canid lineage likely descended from a now-extinct population of Eurasian grey wolves [1]. Multiple approaches have been used to explore the genetic history and diversity of dogs, a question which is complicated by long term population structure and both ancient and modern gene flow among dogs and wolves [2]. The different genomic compartments which have unique patterns of inheritance (i.e. autosomes, mitochondria, and the sex chromosomes) have each revealed

\footnotetext{
* Correspondence: jmkidd@umich.edu

'Department of Human Genetics, University of Michigan Medical School, Ann Arbor, MI, USA

${ }^{3}$ Department of Computational Medicine \& Bioinformatics, University of Michigan Medical School, Ann Arbor, MI, USA

Full list of author information is available at the end of the article
}

novel, and sometimes conflicting, aspects of dog evolution [3-7]. The Y-chromosome and the mitochondria are present as single, largely non-recombining haplotypes and each thus represents a single locus sampled from the evolutionary history of a species. Due to their inheritance patterns, the Y-chromosome and the mitochondria have a reduced effective population size and are more sensitive to the impact of genetic drift. Nonetheless, the single trees represented by these uniparentally inherited loci can be informative for reconstructing population history [8]. Due to their increased copy number per cell, mitochondrial DNA is more easily obtained from ancient samples than nuclear DNA. Thus, mitochondrial analysis has been a key feature of studies

(c) The Author(s). 2018 Open Access This article is distributed under the terms of the Creative Commons Attribution 4.0 International License (http://creativecommons.org/licenses/by/4.0/), which permits unrestricted use, distribution, and reproduction in any medium, provided you give appropriate credit to the original author(s) and the source, provide a link to the Creative Commons license, and indicate if changes were made. The Creative Commons Public Domain Dedication waiver (http://creativecommons.org/publicdomain/zero/1.0/) applies to the data made available in this article, unless otherwise stated. 
involving ancient DNA and have revealed important insights into the evolutionary history of canids $[6,9]$.

Canid mitochondrial phylogenies show that dogs and wolves are not reciprocally monophyletic [6]. The mitochondrial tree contains four deeply rooted clades encompassing dogs and many grey wolf groups. These four clades form the basis of dog mitochondrial haplogroup assignment, known as haplogroups A-D [6, 10]. The time of the most recent common ancestor (TMRCA) of haplogroups A-D significantly predates estimates for domestication based on archeological and genetic evidence $[1,4$, $6,9,11,12]$. Instead, these clades may represent variation present among the founding population of the dog lineage or the results of wolf introgressions into dog populations. The relative frequencies of mitochondria haplogroups are not stable over time, with changes reflecting processes such as drift, migration, and population growth. Although the mitochondria A and B haplogroups are most common in contemporary European dogs, surveys of ancient samples indicate that the majority of ancient European dogs carried the $\mathrm{C}$ or $\mathrm{D}$ mitochondrial haplotype. This apparent turnover in mitochondrial haplogroups may reflect the migration of a distinct dog population into Europe over the past 15,000 years [9].

Relative to the mitochondria, comparatively little is known about the Y-chromosome haplogroup diversity present among ancient dogs in Europe. Similar to the mitochondria, studies of contemporary samples show that the Y-chromosome tree is characterized by deep splits among dogs [7]. A study of Y-chromosome sequence based on a partial assembly (14.4 kb in length) collected from 151 dogs revealed five haplogroups [13]. Larger studies of hundreds of samples using genotyping arrays designed to include Y-chromosome markers show a high diversity of Y-chromosome haplotypes across Africa, India, Central Asia and Southwest Asia [3].

Hundreds of dog and wolf samples have been sequenced using short-read technology, but there has been limited focus on dog Y-chromosome evolution. In part, this is because the presence of highly duplicated sequences, known as amplicons necessitates special care in variant calling. Recently, a canine Y-chromosome reference was sequenced using 454-sequencing applied to pools of BAC clones [14]. This sequence includes a $1 \mathrm{Mb}$ region of amplicon sequence which includes multiple copies of $S R Y$, duplication of which has also been observed in pigs and rabbits $[15,16]$. Here, we utilize this Ychromosome reference along with whole genome sequence data from 118 samples to determine the Y-chromosome haplotype diversity found among contemporary and ancient dogs. First, we utilize publically available short-read data to identify regions of the Y-chromosome amendable to variant identification. Using this map of callable regions, we resolve the Y-chromosome phylogeny and define SNVs diagnostic to each haplogroup using publically available genome sequence data from 118 samples. We then assess the geographic distribution of each haplogroup among modern dogs based on autosomal ancestry inferred using genome sequence data from 104 male dogs. Finally, we interrogate the previously published sequences of three ancient dog genomes to provide an initial assessment of Y-chromosome diversity in Europe during the Neolithic.

\section{Results \\ Identifying Y-chromosome segments suitable for SNV calling}

We mapped Illumina short read libraries from a diverse collection of 118 publically available canid genomes to the $\mathrm{Li}$ et al. canine Y-chromosome assembly (Additional file 1: Table S1). This sample set includes 1 coyote [17], 13 wolves [4, 17-20], 30 village dogs including samples from India, Portugal, Nigeria, and China [4, 21, 22], and 74 breed dogs [4, 19-25]. Based on metrics such as raw read depth, MQ0-to-depth ratio, and the apparent presence of heterozygous variant calls, we identified 484,924 positions on the $\mathrm{Y}$ assembly amendable to variant identification using short-sequencing reads (Fig. 1, Additional file 2: Table S2) [26, 27]. As expected, the regions that pass this callability mask are located outside of the boundaries of amplicons identified by a dotplot analysis of the existing assembly (Additional file 3: Figure S1, Additional file 4: Table S3). Since amplicons may be dynamic over evolutionary time, we also constructed a callability mask using reads only from the coyote sample (Additional file 5: Figure S2). The coyote mask is nearly identical to that obtained from the combined canid dataset, suggesting that divergence between coyote and dog will not lead to largescale errors in read mapping for this analysis. In total, we identified 1221 biallelic Y-chromosome SNVs across the 118 analyzed samples.

\section{Canid Y-chromosome phylogeny}

To assign haplogroup labels and aid comparisons with prior studies, we remapped 151 markers present on the 170 K Illumina HD Canine SNP Array to the Li et al. Ychromosome assembly [3]. This included seven derived alleles specific to the HG8 haplogroup, eight specific to the HG6 haplogroup, and two specific to the HG23 haplogroup. We reclassified some haplotypes, and refer to the four HG1-3 haplotypes Hb.1, H27, H5b and H5a collectively as HG27. The $170 \mathrm{~K}$ Illumina HD Canine SNP Array contains a single diagnostic site specific to the HG27 haplogroup as well as one site specific to HG1-3. Additionally, a variant diagnostic for the HG9 haplogroup [7] was remapped to the Li et al. reference and included in the present analysis. Using genotypes at these sites, we assigned 109 Y-chromosomes to one of these six major dog haplogroups. Most of the Y- 


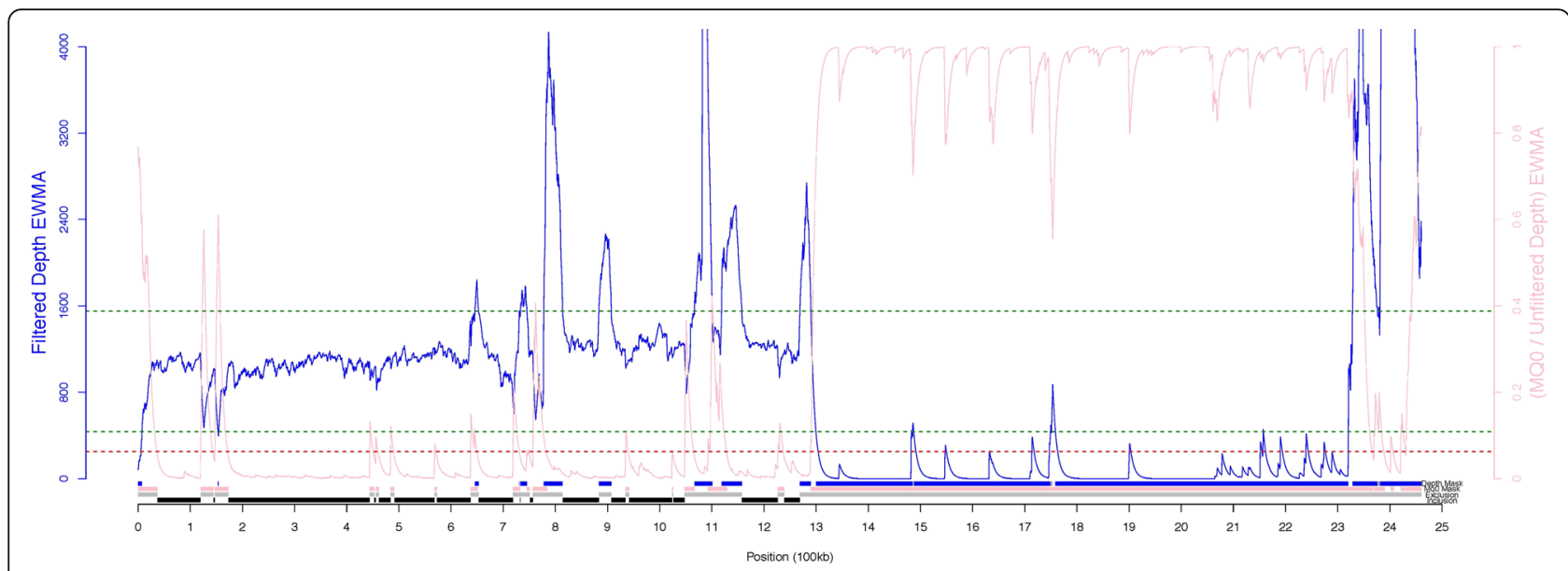

Fig. 1 Regional callability mask to identify regions suitable for variant calling Exponentially weighted moving averages (EWMA) of read depth (blue line) and the mq0/unfiltered depth ratio (pink line) are plotted along the Y-chromosome sequence (KP081776.1). The dashed lines represent maximum and minimum thresholds for filtered depth (green) and a maximum threshold for the mq0 ratio (red). Colored bars below the plot indicate regions masked by the depth filter (blue), masked by the mq0 ratio filter (pink), excluded from the analysis (grey), and included (black) prior to site-level filtering

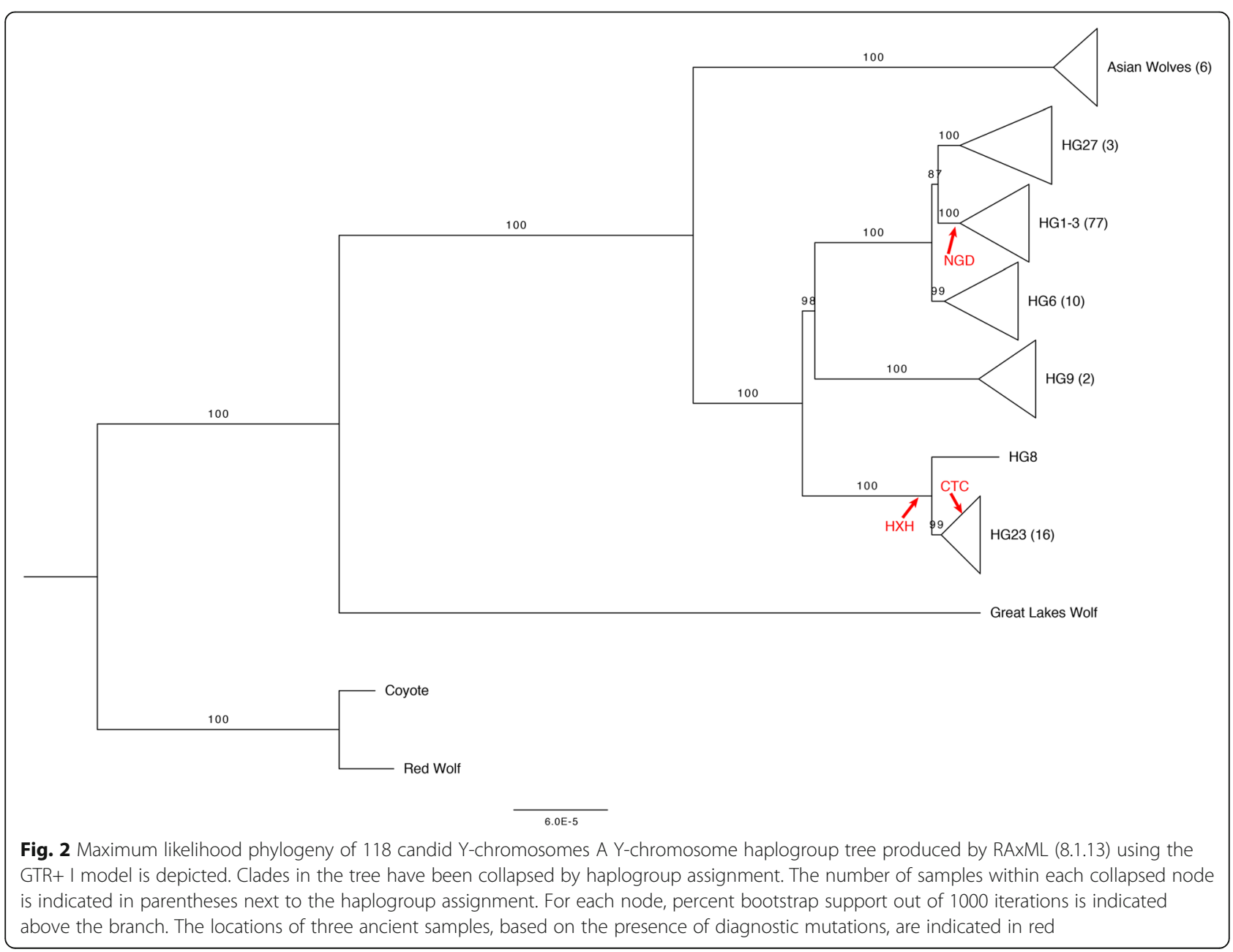


chromosomes in our dataset belong to the HG1-3 clade $(n=77)$, followed by HG23 $(n=16)$, HG6 $(n=10)$, HG27 $(n=3)$, HG9 $(n=2)$, and HG8 $(\mathrm{n}=1)$. Each haplogroup is represented by distinct nodes in maximum likelihood phylogeny constructed from the full set of 1221 SNVs discovered in the resequencing data (Fig. 2). The structure of the phylogeny reveals that HG1-3, HG27 and their sister clade HG6 share a common ancestor that emerged long after their split from HG9. Haplogroup HG8, predominantly found in Africa and the Middle East, is represented by one of the two Nigerian village dogs included in this study, and is related to broadly distributed HG23 haplogroup [3].

Five gray wolf samples were carriers of dog haplogroups (HG1-3 clade $(n=1)$; HG23 $(n=2)$; HG6 $(n=1)$; HG9 $(\mathrm{n}=1)$ ) and are often represented as deep branches within their respective clades (Additional file 6: Figure S3). Recent gene flow or admixture is one potential explanation for the presence of dog haplogroups in these wolves. We performed a model based ancestry analysis of the included dogs and wolves based on autosomal genetic variation discovered from the whole genome sequencing data. This analysis did not identify a substantial degree of dog ancestry in these wolf samples (Additional file 7: Figure S4); however, the Nigerian village dog NG03, which is assigned to the HG9 haplogroup along with a Chinese wolf sample, has detectable wolf ancestry (Additional file 7: Figure S4, $K=3$ ). Similar levels of wolf ancestry were also detected in other village dogs from Nigeria, India, and Portugal.

Additionally, we observed a distinct wolf patriline found in six samples from the Shanxi, Qinghai, Xinjiang, and Tibetan regions. This branch, which we refer to here as Asian Wolves, is comparably deep with respect to the tree and is consistent with a split prior to the formation of the dog haplogroups. However, we note that other Asian wolves carry Y-chromosomes which cluster with the dog HG9 (a Xinjiang wolf) or HG23 haplogroups (a Xinjiang and an Indian wolf).

The red wolf-coyote clade and the Great Lakes wolf represent the deepest branches in the canid phylogeny. Consistent with previous analyses of canid Ychromosomes and mitochondria [6, 7], the coyote Ychromosome is divergent from the grey wolf-dog clade. Instead, the coyote shares a clade with the red wolf, a group known to contain a high amount of coyote admixture $[28,29]$. The single male Great Lakes wolf (a sample from Minnesota [19] carries a strikingly divergent Ychromosome, with 199 derived alleles (16\% of the total SNVs) unique to this Y-chromosome.

To infer the TMRCA of Y-chromosome clades, we used the Bayesian Markov chain Monte Carlo approach implemented in BEAST, which yielded the same tree topology as obtained with RAxML (Fig. 2). We calibrated our estimates by setting a prior TMRCA at the root of the phylogeny as 1.5 million years ago (mya) based on a previous estimate of the dog/wolf-coyote divergence time [30, 31]. Based on relaxed and strict molecular clock models, we find that haplogroup TMRCAs range from $\sim 70,000$ years (HG23) to $\sim 159,000$ years (HG6) (Table 1). On average, we find 362.81 (s.d. = 8.63) substitutions between dog/wolves and coyote. From this estimate, a naïve counting approach yields a Y-chromosome substitution rate at $2.49 \times 10^{-10}$ substitutions per site per year, a value slightly smaller than that obtained from a strict $\left(2.86 \times 10^{-10}\right.$ substitutions per site per year, 95\% HPD: $2.00-3.679 \times 10^{-10}$ ) or relaxed molecular clock model $\left(3.07 \times 10^{-10}\right.$ substitutions per site per year, 95\% HPD: $\left.1.24-5.15 \times 10^{-10}\right)$.

\section{Autosomal genetic ancestry and Y-chromosome Haplogroups}

Next, we assessed the relationship between Ychromosome haplogroups and genetic ancestry using principal components analysis. We defined ancestry based on variation at the autosomal positons present on the 170 K Illumina HD Canine SNP Array. First, we defined broad patterns of geographic diversity based on 499 village dogs (Fig. 3a) which have known ancestry and have been previously genotyped at these positions by Shannon et al. [3]. Next, we used the whole genome sequencing data to genotype the 104 male dogs used in this study at these autosomal positions. The resulting genotypes were then projected onto the PC space defined by the 499 village dogs (Fig. 3b). Based on this assessment of autosomal ancestry, we confirm that the breed dogs carrying the HG13 haplogroup mostly cluster with village dogs from Europe and the Americas with some exceptions: a Husky with the HG1-3 haplogroup clustered with the Arctic samples and six HG1-3 Chinese Village dogs from the Diqing, Lijiang, and Yingjiang regions, displayed Asian ancestry. In contrast, the HG23 haplogroup is found in samples with a broader distribution of ancestry, including village dogs from China that show the expected Asian autosomal ancestry, an Indian Village dog, as well breed dogs with Middle Eastern/Indian ancestry (Afghan Hound, Saluki, and Sloughi) as well as Tibetan Terriers whose ancestry projects near European/American center. The HG6 samples in our study include an Indian village dog, which clusters with Indian reference samples as well as Tibetan Mastiffs and Chinese village dogs that display Asian ancestry. The three HG27 samples (a Korean Jindo, Tibetan Mastiff, and Shiba Inu) have ancestry which appears intermediate between village dogs from Central Asia and Vietnam. As previously mentioned, the HG8 and HG9 haplogroups are each found in a single village dog from Nigeria, each of which projects along the variation found in Africa. 
Table 1 TMRCA Values

\begin{tabular}{|c|c|c|c|c|c|}
\hline Branch & $\begin{array}{l}\text { Diagnostic } \\
\text { Mutations }\end{array}$ & TMRCA $^{\text {a }}$ (Relaxed Clock) & ESS $^{b}$ & TMRCA $^{\text {a }}$ (Strict Clock) & ESS $^{b}$ \\
\hline HG1-3; HG27; HG6; HG9; HG8; HG23; AW; GLW; & 150 & $1.4938[0.6896,2.6717]$ & 1740 & $1.459[1.0653,1.8996]$ & 11,096 \\
\hline HG1-3; HG27; HG6; HG9; HG8; HG23; AW; & 109 & $0.7676[0.3031,1.3928]$ & 1139 & $0.77[0.5463,1.0179]$ & 3436 \\
\hline HG1-3; HG27; HG6; HG9; HG8; HG23; & 34 & $0.4772[0.1917,0.8899]$ & 934 & $0.4792[0.3336,0.6367]$ & 5806 \\
\hline HG1-3; HG27; HG6; HG9; & 4 & $0.4373[0.173,0.8129]$ & 930 & $0.446[0.3136,0.5989]$ & 5763 \\
\hline HG1-3; HG27; HG6; & 33 & $0.2019[0.0803,0.3651]$ & 1021 & $0.1948[0.1332,0.2631]$ & 4671 \\
\hline HG1-3; HG27; & 2 & $0.1805[0.073,0.3285]$ & 1024 & $0.1765[0.1201,0.239]$ & 5466 \\
\hline HG8; HG23; & 40 & $0.1139[0.0387,0.2173]$ & 1081 & $0.1172[0.0702,0.1675]$ & 6907 \\
\hline $\mathrm{HG} 1-3$ & 7 & $0.0975[0.0366,0.1811]$ & 1113 & $0.099[0.0614,0.1395]$ & 5466 \\
\hline $\mathrm{HG} 27$ & 7 & $0.138[0.0525,0.2543]$ & 1023 & $0.1387[0.0917,0.1908]$ & 5359 \\
\hline HG6 & 4 & $0.1599[0.0642,0.2962]$ & 1090 & $0.1578[0.1045,0.2171]$ & 5807 \\
\hline HG9 & 50 & $0.0923[0.0258,0.184]$ & 1138 & $0.0907[0.0509,0.1359]$ & 7075 \\
\hline HG23 & 2 & $0.0715[0.0246,0.1352]$ & 1027 & $0.0708[0.0418,0.1045]$ & 3861 \\
\hline HG8 & 20 & NA & NA & NA & \\
\hline Asian Wolves & 112 & $0.0636[0.0202,0.1240]$ & 919 & $0.0562[0.0308,0.0838]$ & 3646 \\
\hline Great Lakes Wolf & 199 & NA & NA & NA & \\
\hline Incompatible & 21 & NA & NA & NA & \\
\hline
\end{tabular}

aTMRCA in millions of years, with $95 \%$ highest posterior density interval

${ }^{\text {b}}$ Estimated effective sample size

\section{Y-chromosome variation in three ancient dogs}

To offer an initial depiction of Y-chromosome diversity among ancient dogs, we determined Y-chromosome haplotypes for three recently published ancient samples from Europe $[9,11]$. This includes an ancient dog dated to $\sim 4800$ calendar years before the present found in the Newgrange grave complex in Ireland (abbreviated NGD), a sample from the early Neolithic site of Herxheim, Germany, dated to $\sim 7000$ years ago (abbreviated HXH), and a dog from the Cherry Tree Cave in Bavaria, Germany estimated to be $\sim 4700$ years old (abbreviated CTC). All three ancient samples were previously reported as being male. We assigned $\mathrm{Y}$ haplogroups using the diagnostic alleles we identified (Additional file 6: Figure S3). CTC and $\mathrm{HXH}$ both had the derived alleles at all the HG8-HG23 diagnostic sites (21 sites passing quality filters in both samples). Unfortunately, sites specifically diagnostic for the HG23 or HG8 clades were not callable in these two
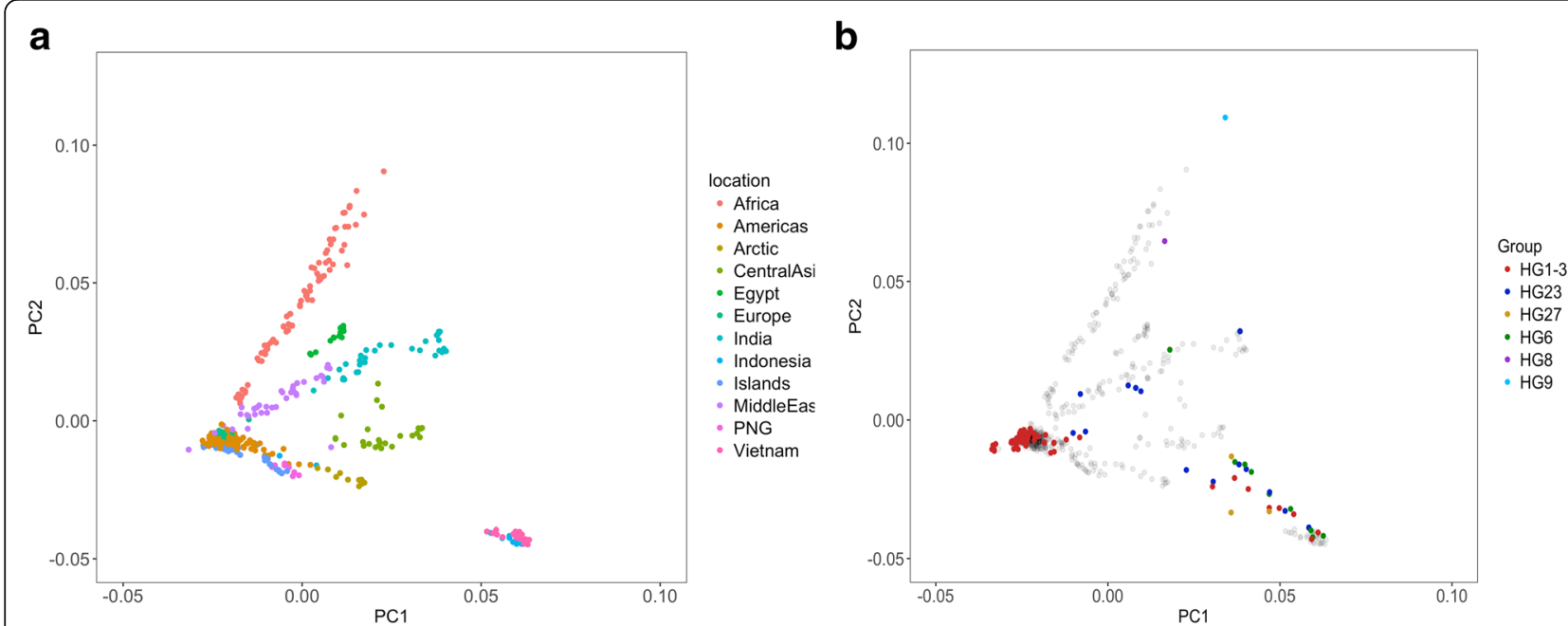

Fig. 3 The relationship between autosomal ancestry and Y-chromosome haplogroups Major groupings of canine ancestry are shown based on a principal components analysis of autosomal markers from 499 village dogs from Shannon et al. $\mathbf{a}$. The geographic origin of each sample is indicated by color. The 104 male dogs used in this study are projected onto the resulting principal components and colored based on haplogroup (b). Village dogs from (a) are shown as transparent dots in (b) 
samples. However, CTC carried 2 of 4 callable derived alleles that were unique to the India wolf leaf, a HG23 haplotype. The NGD Y-chromosome belongs to the HG1-3 haplogroup (7 of 7 callable derived alleles). Unlike CTC, it did not carry any diagnostic alleles that matched any contemporary modern dogs or wolves within the HG1-3 haplogroup.

\section{Discussion}

In this study we present an analysis of Y-chromosome sequence diversity based on mapping whole genome sequence data from 118 canids to a published canine Ychromosome assembly. Despite the incomplete representation of Y-chromosome amplicons, the published reference provides a critical resource for phylogenetic analysis of Y-chromosome sequence variation. Our data supports the Y-chromosome haplogroup definitions previously reported [3, 7]. Relative to the Y-chromosome network presented by Shannon et al., we find that HG27 is divergent from HG1-3 and we suggest that it should should be considered a unique haplogroup.

Using the variation discovered from sequence data, we applied a Bayesian MCMC approach to estimate TMRCAs for each haplotype group. Our estimated Ychromosome mutation rate $\left(3.07 \times 10^{-10}\right.$ substitutions per site per year, relaxed clock model) falls within the range of a previous estimate by Ding et al. who used a similar calibration and estimate $1.35 \times 10^{-10}-4.31 \times 10^{-}$

${ }^{10}$ substitutions per site per year [7]. The TMRCAs we estimated are substantially older than mitochondria phylogenies calibrated with tip dates of ancient samples, [6] which report clade-specific TMRCAs $<25,000$ years ago. We note that our Y-chromosome TMRCA estimates are extremely sensitive to our assumptions about the age of the root of the tree and should be interpreted with caution due to the uncertainty in this single calibration point. However, the relative ages of the branches and the chronological order of haplogroup divergences are more robust than the absolute estimated dates.

In general, the relationships between Y-chromosome haplogroups and autosomal ancestry we report are very similar to the relationships described in Shannon et al. [3] As noted earlier, our dataset includes a subset of wolves with Y-chromosomes assigned to a dog Yhaplogroup. However, ADMIXTURE analysis does not indicate substantial recent dog ancestry in these samples, suggesting that their placement on the Y-chromosome phylogeny reflects variation in Y-chromosome haplotypes that was present in the ancestral population and therefore predates the domestication process or is the result of ancient introgression events whose signature of autosomal ancestry has been diluted.

The high divergence of the Y-chromosome from a single a Great Lakes wolf is unexpected. The Great Lakes wolves are hypothesized to be a long-standing ecotype of the grey wolf that persisted in the presence of genetic introgression from coyote and other grey wolves [29, 32]. A previous study of Y-chromosome microsatellite data from Great Lakes wolves did not find evidence of a unique grouping, instead finding that some Great Lakes wolves carried Y-chromosomes that clustered with coyotes while others carried haplogroups that clustered with other grey wolves [32]. The retention of such a deeply diverged lineage may be the result of strong population structure in the past history of Great Lakes wolves. Further interpretation will require a more diverse collection of Y-chromosome sequences from additional coyotes and wolves.

Using our expanded set of clade-specific mutations, we assessed the Y-chromosome haplogroups found in three recently published ancient canine genomes from Europe. The two samples from Germany, CTC and HXH, belonged to the HG8-HG23 clade which is common in contemporary dogs from Asia and the Middle East. Previously published analysis of the autosomal genome of CTC indicated shared ancestry between CTC and modern wolves that are now found in India and Iran. Consistent with this proposed ancestry, our analysis shows that the CTC Y-chromosome shares derived alleles with an Indian wolf [11]. In contrast, NGD, a sample from Ireland and a near contemporary of CTC, belongs to the HG1-3 haplogroup which is mostly found in modern European dogs. This Y-chromosome data, which shows that at least two Y-chromosome haplogroups were present among European dogs during the Neolithic, supports the existence of long-lasting population structure among European dogs.

\section{Conclusions}

Using sequencing data, we find that the estimated TMRCA of dog Y haplogroups predates dog domestication. We further reveal the placement of several wolf Ychromosomes within deep branches of dog haplogroup clades. Using an expanded set of mutations diagnostic for each haplogroup, we find that distinct $Y$ haplogroups were present in Europe during the Neolithic and that CTC, a $~ 4700$ year old ancient dog from Germany has a Y-chromosome that shares diagnostic alleles with wolves found in India.

\section{Methods}

\section{Sample selection and Y-chromosome data processing}

The canid short-read sequencing data used in this analysis are available from the NCBI Sequence Read Archive (SRA; Additional file 1: Table S1). Sequencing runs from each experiment were independently aligned to a canine reference genome that included both the CanFam3.1 sequence and the canine Y-chromosome assembly from Li. Et al. (KP081776.1) [14]. We note that KP081776.1 is present in the opposite chromosome orientation from 
that typically employed. Read alignment was performed with bwa mem (version 0.7.13) using -t 4 -M flags [33]. Sample identity across multiple sequencing runs was confirmed by assessment of identity-by-descent at SNPs included on the $170 \mathrm{~K}$ Illumina HD Canine SNP array. IBD across all runs was calculated using the Plink -genome function [34]. We removed samples with sequencing runs ( $>100,000$ reads) that displayed a low level of relatedness across runs (pi-hat $<0.90$ ) to avoid merging runs derived from different biological samples. Once runs within each sample were merged, we sorted and marked duplicates of the alignment files using Picard (version 2.3.0). Base quality values in BAM files were recalibrated and variants were then calculated using the GATK haplotype caller (version 3.5-0) [35].

To identify male samples for inclusion in our study population, we calculated each sample's average autosome to X-chromosome (A/X) sequencing depth. We included samples that had an $\mathrm{A} / \mathrm{X}$ sequencing depth ratio $>1.85$ and an autosomal coverage $>10 \times$. The final sample set contained 118 samples including 1 coyote [17], 13 wolves [4, 17-20], 30 village dogs including samples from India, Portugal, Nigeria, and China $[4,21$, $22]$, and 74 breed dogs $[4,19-25]$. We note that this set includes two Afghan Hounds which are clones of each other and therefore have identical Y-chromosome sequences [25]. We next recalled Y-chromosome genotypes in the male samples using the GATK haplotype caller using the EMIT_ALL_SITES flag. Following methods previously used for identifying repetitive sequence unsuitable for read mapping in primate Ychromosomes, we used mapping and depth statistics from the VCF info field to identify callable regions on the canine Y-chromosome (Fig. 1) [26, 27]. Once a callable region was identified, we applied site level filtering to the remaining sequence: dropping maximum likelihood heterozygotes, missing sites, and positions with an MQ0/ raw depth ratio $>0.10$. A second depth filter was then applied to remove positions with extreme sequencing depths (median depth \pm 3 M.A.D.). We also removed positions that were within $5 \mathrm{bp}$ of GATK called indels.

Three ancient canine samples previously identified as male (NGD, CTC, and HXH dogs) were aligned to our custom canine reference using the same procedure as described previously $[9,11]$. In short, the damage patterns identified with mapDamage were adjusted into the confidence of the variant calling [36,37]. We limited our analysis of ancient samples to positions with a minimum read depth of 4; genotype quality score of 30; map quality score of 15 ; and base quality score of 15 .

\section{Haplogroup assignment}

We assigned Y-chromosome haplogroups using the definitions from Shannon et al. based on the 207 Y-chromosome polymorphic variants captured on the $170 \mathrm{~K}$ Illumina HD Canine SNP Array [3]. Since the $170 \mathrm{~K}$ Illumina HD Canine SNP Array Y-chromosome probes were designed on a less complete Y-chromosome assembly (chrY_nonPAB), we remapped the SNP locations to the Li et al. assembly [14]. First, we extracted $+/-50$ bp of sequence from each SNP position on the chrY_nonPAB assembly and determined the location of each $101 \mathrm{bp}$ long fragment on the Li et al. Y-chromosome assembly using blat [38]. We filtered sequences that mapped to multiple regions at an identity $>0$. 99, had ambiguous orientations, or did not pass the filtering criteria mentioned above.

\section{Phylogenetic analysis}

Maximum likelihood (ML) trees were reconstructed from the Y-chromosome sequence using RAxML (8.1.13) with 1000 bootstrap replicates [39]. The GTR+ I model was identified by jModelTest 2 as the best fitting substitution model of 12 candidates as determined by the lowest AIC [40]. We estimated the time of the most recent common ancestor (TMRCA) for branches of interest using the MCMC method BEAST using both a strict and relaxed molecular clock with a log-normal prior distribution on the dog-coyote TMRCA [41, 42]. We merged two independent BEAST runs performed for 10,000,000 iterations with sampling every 1000 iterations. Convergence of MCMC chains was assessed with Tracer and analysis of consensus plotted with FigTree yielded the same tree topology as found with RAxML [42].

We assigned SNVs in our call set to branches within this phylogeny using the ETE toolkit in python [43]. For a given SNV, we identify all leaves in the tree carrying the genotype and iteratively checked deeper nodes to test if all samples are also carriers. This continued with recursion until the deepest node of genotype universality was identified. If a single top node is identified for a variant, we consider that variant diagnostic for the given node. If multiple top nodes are identified, the variant is called as potentially recurrent and is incompatible with the phylogenetic tree.

\section{Ancestry and geography}

Autosomal genetic ancestry was visualized via a principal components analysis (PCA) using the smartPCA program from eigensoft version 3.0 [44]. For the PCA, we limited variants to those present on the $170 \mathrm{~K}$ Illumina HD Canine SNP Array so that the larger collection of samples from Shannon et al. [3] could be included. Here, ancestry was first estimated using the 499 village dogs from Shannon et al. [3] as this sample set more completely represents global dog genetic diversity than collections of breed dogs, and the male dogs $(n=104)$ from our study were subsequently projected onto the resulting components. The proportion of wolf and dog admixture 
for each dog or wolf sample was estimated using the program ADMIXTURE [45]. As input, we used autosomal genotypes from the whole genome short-read data called by GATK HaplotypeCaller. Variant quality score recalibration (VQSR) was applied to the candidate callset using the Illumina $170 \mathrm{~K}$ Illumina HD Canine SNP Array as training and retaining only those SNPs that pass the 99.0 tranche. The VCF file was reformatted into a binary plink file, to which we applied quality control and LD pruning. We removed individual samples $(n=30)$ selected at random from related pairs (pi_hat $>0.125$ ). Variants were filtered with a minor allele frequency $<0.01$ and/or call rate $<0.99$. We pruned variants in strong $L D$ by using the indep-pairwise command in plink with the following parameters: $50 \mathrm{~kb}$ window size, 10 variant step size, and an $\mathrm{r}^{2}$ threshold of 0.1 . For admixture analyses, we modeled $K(\mathrm{k}=2-5)$ ancestral populations with ADMIXURE and plotted the estimated proportion of ancestry of each sample using $\mathrm{R}$. Three independent runs were performed for each value of $\mathrm{K}$. The smallest cross validation error was obtained for $K=2$ in this sample set, (mean of 0.365 versus mean $\mathrm{CV}$-error of 0.368 for $\mathrm{K}=3$ ), however the results for $\mathrm{K}=3$ better correspond to the expected diversity of village and breed dogs.

\section{Additional files}

\section{Additional file 1: Table S1. SRA Sample IDs. (XLS $77 \mathrm{~kb}$ ) \\ Additional file 2: Table S2. Summary of positions passing quality filters. (PDF $102 \mathrm{~kb}$ )}

Additional file 3: Figure S1. Structure and annotation of the dog Ychromosome (KP081776.1) amplicon region (chrY:1,200,000-2,440,580) A self-alignment of the dog Y-chromosome amplicon sequence for visualization of palindrome repeat sequences is presented (A). The three repeat families are color coded in purple, pink, and blue. The annotated sequence with amplicon genes (black) is represented as a UCSC browser track (B). Individual palindrome arms are numbered while palindrome spacers are lettered. (PDF $2240 \mathrm{~kb}$ )

Additional file 4: Table S3. Amplicon coordinates. (XLS $32 \mathrm{~kb}$ )

Additional file 5: Figure S2. Coyote read depth and MQO ratio by position An exponentially weighted moving averages (EWMA) of read depth (blue line) and the mq0/unfiltered depth ratio (pink line) are plotted along the Y-chromosome sequence for the coyote sample. (PNG $164 \mathrm{~kb}$ )

Additional file 6: Figure S3: Individual dog and wolf haplogroup topologies from RAxML tree A. HG1-3, B. HG6 C. HG23-HG8, D. HG27, E. HG9, F. Asian Wolves. Bootstrap support is indicated above branches. The names of wolves and dogs are colored in red and black, respectively. The placement of the three ancient dogs, CTC, HXH, and NGD are indicated as triangles above the most exterior node or leaf where diagnostic mutations indicated their membership. Most bootstrap values from HG13 are not shown due to space limitations. (PDF $334 \mathrm{~kb}$ )

Additional file 7: Figure S4. Autosomal admixture amongst breed dogs, village dogs and wolves. Barplots of ancestry proportions estimated by ADMIXTURE are shown for $\mathrm{K}$ values $2-5$. Breed dogs, village dogs, and wolves are grouped and ordered from left to right. (PDF $1138 \mathrm{~kb}$ )

Additional file 8: Y-chromosome SNV genotypes. (GZ 35059 kb)

Additional file 9: Table S4. Diagnostic mutations for each major branch. (XLS $132 \mathrm{~kb}$ )

\section{Abbreviations}

GATK: Genome Analysis Tool Kit; MCMC: Markov chain Monte Carlo; PCA: principal component analysis; SNP: single nucleotide polymorphism; SNV: single nucleotide variant; TMRCA: time to the most recent common ancestor

\section{Acknowledgements}

We thank Shiya Song and Amanda L. Pendleton for assistance with sample curation and Sarah Emery and Lauren O'Connor for advice on reference structure assessment.

\section{Funding}

This work was supported by grant R01GM103961 from the National Institutes of Health. The funding agency had no role in the design, analysis or interpretation of the study.

\section{Availability of data and materials}

The datasets analysed during the current study are available in the Sequence Read Archive (SRA, https://www.ncbi.nlm.nih.gov/sra). SRA accession IDs for the analyzed samples are given in Additional file 1: Table S1. Additional data generated or analyzed during this study are included in this published article and its supplementary information files, including the underling SNV genotypes (Additional file 8), and the assignment of each position to specific branches on the inferred phylogeny (Additional file 9: Table S4).

\section{Authors' contributions}

MTO and JMK designed the study. MTO, AM, and KRV processed data and performed analyses. MTO and JMK wrote the manuscript with input from all authors. All authors have read and approved the manuscript.

Ethics approval and consent to participate

Not applicable.

\section{Competing interests}

The authors declare that they have no competing interests.

\section{Publisher's Note}

Springer Nature remains neutral with regard to jurisdictional claims in published maps and institutional affiliations.

\section{Author details}

'Department of Human Genetics, University of Michigan Medical School, Ann Arbor, MI, USA. ${ }^{2}$ Department of Ecology and Evolution, Stony Brook University, Stony Brook, New York, USA. ${ }^{3}$ Department of Computational Medicine \& Bioinformatics, University of Michigan Medical School, Ann Arbor, $\mathrm{MI}$, USA.

Received: 22 November 2017 Accepted: 2 May 2018

Published online: 10 May 2018

\section{References}

1. Freedman AH, Gronau I, Schweizer RM, Ortega-Del Vecchyo D, Han E, Silva PM, Galaverni M, Fan Z, Marx P, Lorente-Galdos B, et al. Genome sequencing highlights the dynamic early history of dogs. PLoS Genet. 2014; 10(1):e1004016.

2. Larson G, Bradley DG. How much is that in dog years? The advent of canine population genomics. PLoS Genet. 2014;10(1):e1004093.

3. Shannon LM, Boyko RH, Castelhano M, Corey E, Hayward JJ, McLean C, White ME, Abi Said M, Anita BA, Bondjengo Nl, et al. Genetic structure in village dogs reveals a central Asian domestication origin. Proc Natl Acad Sci U S A. 2015;112(44):13639-44.

4. Wang GD, Zhai W, Yang HC, Wang L, Zhong L, Liu YH, Fan RX, Yin TT, Zhu $C L$, Poyarkov AD, et al. Out of southern East Asia: the natural history of domestic dogs across the world. Cell Res. 2016;26(1):21-33.

5. Pang JF, Kluetsch C, Zou XJ, Zhang AB, Luo LY, Angleby H, Ardalan A, Ekstrom C, Skollermo A, Lundeberg J, et al. mtDNA data indicate a single origin for dogs south of Yangtze River, less than 16,300 years ago, from numerous wolves. Mol Biol Evol. 2009;26(12):2849-64.

6. Thalmann O, Shapiro B, Cui P, Schuenemann VJ, Sawyer SK, Greenfield DL, Germonpre MB, Sablin MV, Lopez-Giraldez F, Domingo-Roura X, et al. 
Complete mitochondrial genomes of ancient canids suggest a European origin of domestic dogs. Science. 2013;342(6160):871-4.

7. Ding ZL, Oskarsson M, Ardalan A, Angleby H, Dahlgren LG, Tepeli C, Kirkness E, Savolainen P, Zhang YP. Origins of domestic dog in southern East Asia is supported by analysis of Y-chromosome DNA. Heredity (Edinb). 2012;108(5):507-14.

8. Jobling MA, Tyler-Smith C. The human Y chromosome: an evolutionary marker comes of age. Nat Rev Genet. 2003;4(8):598-612.

9. Frantz LA, Mullin VE, Pionnier-Capitan M, Lebrasseur O, Ollivier M, Perri A, Linderholm A, Mattiangeli V, Teasdale MD, Dimopoulos EA, et al. Genomic and archaeological evidence suggest a dual origin of domestic dogs. Science. 2016;352(6290):1228-31.

10. Vila C, Savolainen P, Maldonado JE, Amorim IR, Rice JE, Honeycutt RL, Crandall KA, Lundeberg J, Wayne RK. Multiple and ancient origins of the domestic dog. Science. 1997;276(5319):1687-9.

11. Botigue $L R$, Song $S$, Scheu A, Gopalan S, Pendleton AL, Oetjens M, Taravella AM, Seregely T, Zeeb-Lanz A, Arbogast RM, et al. Ancient European dog genomes reveal continuity since the early Neolithic. Nat Commun. 2017:8:16082

12. Skoglund P, Ersmark E, Palkopoulou E, Dalen L. Ancient wolf genome reveals an early divergence of domestic dog ancestors and admixture into high-latitude breeds. Curr Biol. 2015;25(11):1515-9.

13. Natanaelsson C, Oskarsson MC, Angleby H, Lundeberg J, Kirkness E, Savolainen P. Dog Y chromosomal DNA sequence: identification, sequencing and SNP discovery. BMC Genet. 2006;7:45.

14. Li G, Davis BW, Raudsepp T, Pearks Wilkerson AJ, Mason VC, Ferguson-Smith M, O'Brien PC, Waters PD, Murphy WJ. Comparative analysis of mammalian $Y$ chromosomes illuminates ancestral structure and lineage-specific evolution. Genome Res. 2013;23(9):1486-95.

15. Geraldes A, Rambo T, Wing RA, Ferrand N, Nachman MW. Extensive gene conversion drives the concerted evolution of paralogous copies of the SRY gene in European rabbits. Mol Biol Evol. 2010;27(11):2437-40.

16. Skinner BM, Sargent CA, Churcher C, Hunt T, Herrero J, Loveland JE, Dunn M, Louzada S, Fu B, Chow W, et al. The pig X and Y chromosomes: structure, sequence, and evolution. Genome Res. 2016;26(1):130-9.

17. Fan Z, Silva P, Gronau I, Wang S, Armero AS, Schweizer RM, Ramirez O, Pollinger J, Galaverni M, Ortega Del-Vecchyo D, et al. Worldwide patterns of genomic variation and admixture in gray wolves. Genome Res. 2016;26(2):163-73.

18. Zhang W, Fan Z, Han E, Hou R, Zhang L, Galaverni M, Huang J, Liu H, Silva $\mathrm{P}$, Li P, et al. Hypoxia adaptations in the grey wolf (Canis lupus chanco) from Qinghai-Tibet plateau. PLoS Genet. 2014;10(7):e1004466.

19. Marsden CD, Ortega-Del Vecchyo D, O'Brien DP, Taylor JF, Ramirez O, Vila C, Marques-Bonet T, Schnabel RD, Wayne RK, Lohmueller KE. Bottlenecks and selective sweeps during domestication have increased deleterious genetic variation in dogs. Proc Natl Acad Sci U S A. 2016;113(1):152-7.

20. Decker B, Davis BW, Rimbault M, Long AH, Karlins E, Jagannathan V, Reiman R, Parker HG, Drogemuller C, Corneveaux JJ, et al. Comparison against 186 canid whole-genome sequences reveals survival strategies of an ancient clonally transmissible canine tumor. Genome Res. 2015;25(11):1646-55.

21. Auton A, Rui Li Y, Kidd J, Oliveira K, Nadel J, Holloway JK, Hayward JJ, Cohen $\mathrm{PE}$, Greally JM, Wang J, et al. Genetic recombination is targeted towards gene promoter regions in dogs. PLoS Genet. 2013;9(12):e1003984.

22. Li Y, Wu DD, Boyko AR, Wang GD, Wu SF, Irwin DM, Zhang YP. Population variation revealed high-altitude adaptation of Tibetan mastiffs. Mol Biol Evol. 2014;31(5):1200-5.

23. Kim RN, Kim DS, Choi SH, Yoon BH, Kang A, Nam SH, Kim DW, Kim JJ, Ha $\mathrm{JH}$, Toyoda A, et al. Genome analysis of the domestic dog (Korean Jindo) by massively parallel sequencing. DNA Res. 2012;19(3):275-87.

24. Wang GD, Zhai W, Yang HC, Fan RX, Cao X, Zhong L, Wang L, Liu F, Wu H, Cheng $L G$, et al. The genomics of selection in dogs and the parallel evolution between dogs and humans. Nat Commun. 2013:4:1860.

25. Kim HM, Cho YS, Kim H, Jho S, Son B, Choi JY, Kim S, Lee BC, Bhak J, Jang G. Whole genome comparison of donor and cloned dogs. Sci Rep. 2013;3:2998.

26. Poznik GD, Henn BM, Yee MC, Sliwerska E, Euskirchen GM, Lin AA, Snyder M, Quintana-Murci L, Kidd JM, Underhill PA, et al. Sequencing Y chromosomes resolves discrepancy in time to common ancestor of males versus females. Science. 2013;341(6145):562-5.

27. Oetjens MT, Shen F, Emery SB, Zou Z, Kidd JM. Y-chromosome structural diversity in the bonobo and chimpanzee lineages. Genome Biol Evol. 2016; 8(7):2231-40.
28. vonHoldt BM, Cahill JA, Fan ZX, Gronau I, Robinson J, Pollinger JP, Shapiro B, Wall J, Wayne RK. Whole-genome sequence analysis shows that two endemic species of north American wolf are admixtures of the coyote and gray wolf. Sci Adv. 2016;2(7):e1501714.

29. vonHoldt BM, Pollinger JP, Earl DA, Knowles JC, Boyko AR, Parker H, Geffen E, Pilot M, Jedrzejewski W, Jedrzejewska B, et al. A genome-wide perspective on the evolutionary history of enigmatic wolf-like canids. Genome Res. 2011;21(8):1294-305.

30. Duleba A, Skonieczna K, Bogdanowicz W, Malyarchuk B, Grzybowski T. Complete mitochondrial genome database and standardized classification system for Canis lupus familiaris. Forensic Sci Int Genet. 2015;19:123-9.

31. Perini FA, Russo CA, Schrago CG. The evolution of south American endemic canids: a history of rapid diversification and morphological parallelism. J Evol Biol. 2010;23(2):311-22.

32. Koblmuller S, Nord M, Wayne RK, Leonard JA. Origin and status of the Great Lakes wolf. Mol Ecol. 2009;18(11):2313-26.

33. Li H. Aligning sequence reads, clone sequences and assembly contigs with BWA-MEM. In: ArXiv e-prints, vol. 1303; 2013.

34. Purcell $S$, Neale B, Todd-Brown $K$, Thomas L, Ferreira MA, Bender D, Maller J, Sklar P, de Bakker PI, Daly MJ, et al. PLINK: a tool set for whole-genome association and population-based linkage analyses. Am J Hum Genet. 2007; 81(3):559-75.

35. McKenna A, Hanna M, Banks E, Sivachenko A, Cibulskis K, Kernytsky A, Garimella K, Altshuler D, Gabriel S, Daly M, et al. The genome analysis toolkit: a MapReduce framework for analyzing next-generation DNA sequencing data. Genome Res. 2010;20(9):1297-303.

36. Jonsson H, Ginolhac A, Schubert M, Johnson PL, Orlando L. mapDamage2.0: fast approximate Bayesian estimates of ancient DNA damage parameters. Bioinformatics. 2013;29(13):1682-4.

37. Hofmanova Z, Kreutzer S, Hellenthal G, Sell C, Diekmann Y, Diez-Del-Molino D, van Dorp L, Lopez S, Kousathanas A, Link V, et al. Early farmers from across Europe directly descended from Neolithic Aegeans. Proc Natl Acad Sci U S A. 2016;113(25):6886-91.

38. Kent WJ. BLAT-the BLAST-like alignment tool. Genome Res. 2002;12(4):656-64.

39. Stamatakis A. RAxML version 8: a tool for phylogenetic analysis and postanalysis of large phylogenies. Bioinformatics. 2014;30(9):1312-3.

40. Darriba D, Taboada GL, Doallo R, Posada D. jModelTest 2: more models, new heuristics and parallel computing. Nat Methods. 2012;9(8):772.

41. Drummond AJ, Ho SY, Phillips MJ, Rambaut A. Relaxed phylogenetics and dating with confidence. PLoS Biol. 2006;4(5):e88.

42. Drummond AJ, Suchard MA, Xie D, Rambaut A. Bayesian phylogenetics with BEAUti and the BEAST 1.7. Mol Biol Evol. 2012;29(8):1969-73.

43. Huerta-Cepas J, Dopazo J, Gabaldon T. ETE: a python environment for tree exploration. BMC Bioinformatics. 2010;11:24.

44. Patterson N, Price AL, Reich D. Population structure and eigenanalysis. PLoS Genet. 2006;2(12):e190.

45. Alexander DH, Novembre J, Lange K. Fast model-based estimation of ancestry in unrelated individuals. Genome Res. 2009:19(9):1655-64.

\section{Ready to submit your research? Choose BMC and benefit from:}

- fast, convenient online submission

- thorough peer review by experienced researchers in your field

- rapid publication on acceptance

- support for research data, including large and complex data types

- gold Open Access which fosters wider collaboration and increased citations

- maximum visibility for your research: over $100 \mathrm{M}$ website views per year

At BMC, research is always in progress.

Learn more biomedcentral.com/submissions 